\title{
Surgical Treatment of an Innominate Artery Aneurysm Using Near-Infrared Spectroscopy for Cerebral Monitoring: A Case Report
}

\author{
Byeng Hun Jeon, M.D., Chul Ho Lee, M.D., Chi Hoon Bae, M.D., Jae Seok Jang, M.D., Jun Woo Cho, M.D. \\ Department of Thoracic and Cardiovascular Surgery, Daegu Catholic University School of Medicine, Daegu, Korea
}

\section{ARTICLE INFO}

Received April 14, 2021

Revised May 29, 2021

Accepted June 3, 2021

Corresponding author

Jun Woo Cho

Tel 82-53-650-4566

Fax 82-53-629-6963

E-mail jojunwu1@cu.ac.kr

ORCID

https://orcid.org/0000-0002-0786-9775

\begin{abstract}
Innominate artery aneurysms are challenging for surgeons to treat because of the requirement for brain protection during surgery. In innominate artery aneurysms, the endovascular approach does not require cardiopulmonary bypass, but patients who can be treated using this approach are limited in number, and the long-term results of endovascular treatment are unclear. Here, we report our experience of successfully treating a patient with an innominate artery aneurysm using near-infrared spectroscopy without cardiopulmonary bypass support or hypothermic circulatory arrest.
\end{abstract}

Keywords: Innominate artery, Aneurysm, Near-infrared spectroscopy, Case report

\section{Case report}

Isolated innominate artery aneurysms are rare, but can be fatal. Brain protection is the main concern during surgery, and in some cases, hypothermic circulatory arrest is necessary. Herein, we describe a case of a 71-year-old patient with relevant comorbidities in whom surgical treatment of an innominate artery aneurysm was thought to be life-threatening. An endovascular approach was considered first, but her anatomical status was unsuitable. Surgical treatment was then considered without any cardiopulmonary bypass $(\mathrm{CPB})$ support.

A 71-year-old woman with diabetes, hypertension, and new-onset dialysis dependence presented with fever that had started 5 days earlier. Blood culture revealed persistent bacteremia with methicillin-resistant Staphylococcus aureus, and the fever did not subside. Chest and abdomen computed tomography were performed. A $3-\mathrm{cm}$ aneurysm was detected in the right innominate artery, and a saccular aneurysm was observed in the descending thoracic aorta (Fig. 1). As the left brachio-axillary arteriovenous graft was considered to be the focus of bacteremia, the graft was removed before the correction of the aneurysm. Pus-like discharge was observed in the operative field near the graft,

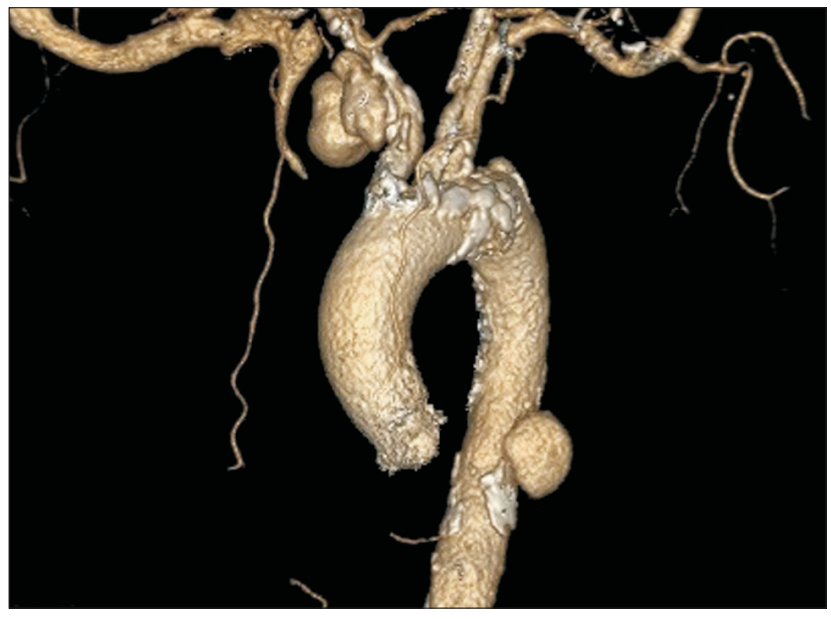

Fig. 1. Preoperative 3-dimensional computed tomography of the aorta. A 5-cm aneurysm was found in the right upper paratracheal space, arising from the innominate artery.

but the culture test of the postoperative graft specimen was negative. Blood culture was performed once a week, and surgery was planned when bacterial cultures were negative at least 3 times. Finally, vancomycin was administered for a total of 8 weeks until surgery, and simultaneous correction of the innominate artery aneurysm and descending 
thoracic aorta aneurysm was planned. The landing zone of the innominate artery aneurysm was unsuitable for endovascular treatment because the proximal and distal parts of the aneurysm had insufficient space to enable stenting and were atherosclerotic, which could increase the risk of stroke. Moreover, surgical resection of the aneurysm was needed because the patient had a right dominant vertebral artery from the innominate artery. The combination of surgical resection of the innominate artery aneurysm with an endovascular approach only for the descending aorta aneurysm was considered the best treatment option for the patient with respect to the operative time, postoperative recurrence, and risk of postoperative complications.

After successfully inserting a stent graft $\left(30 \times 130 \mathrm{~mm}^{2}\right.$; S\&G Biotech, Seongnam, Korea) in the descending thoracic aorta aneurysm through the left femoral artery, the innominate artery aneurysm was resected. The operative field was secured with median full sternotomy and right cervical extension (Fig. 2). Due to concerns about the risk of aneurysm rupture, we chose to prepare for an emergency by performing full sternotomy, rather than using an approach that would provide limited exposure of the surgical field. A Y-graft was created in the operative field using 10and 8-mm Dacron grafts (Hemashield Platinum; Intervascular SAS, La Ciotat, France), and the graft was anastomosed in an end-to-side fashion with the ascending aorta. After clamping the right common carotid artery and the right subclavian artery, the origin of the innominate artery was divided from the aorta using a surgical stapler (Ethicon Endo-Surgery Europe GmbH, Norderstedt, Germany), which is commonly used for the resection of pulmonary vessels and bronchus in general thoracic surgery and gastrointestinal anastomosis in abdominal surgery given its ease of use and time-saving qualities. A stapler was applied

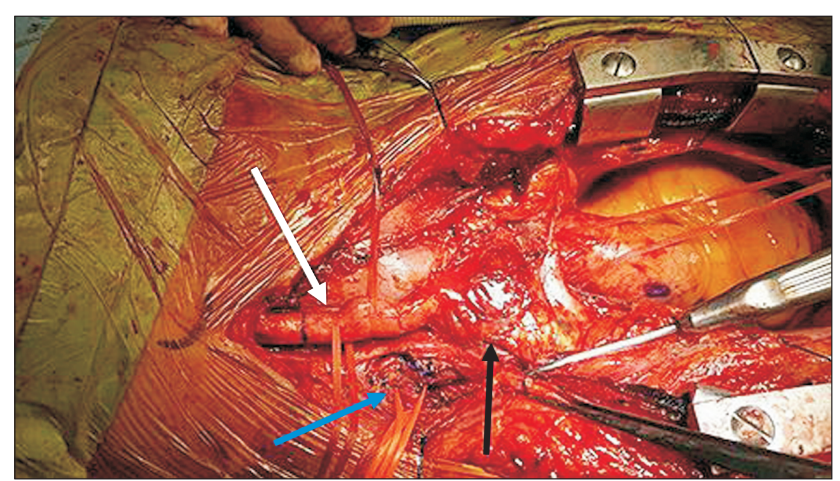

Fig. 2. Operative field of view of full median sternotomy and cervical extension. White and blue arrows show the right common carotid artery and right subclavian artery, respectively. A black arrow shows aneurysmal change of the innominate artery. to healthy tissues on the artery. Near-infrared spectroscopy (NIRS, INVOS 5100CTM; Covidien, Minneapolis, MN, USA) was employed for cerebral monitoring while clamping the right common carotid artery. If the NIRS values had decreased, we would have inserted a shunt between the Y-graft of Dacron and the right common carotid artery to maintain brain perfusion. Fortunately, no significant alteration in the cerebral oximetry values was observed. The Y-graft was anastomosed in an end-to-end fashion with the right common carotid and right subclavian arteries after the resection of the aneurysm (Fig. 3). The right common carotid artery was first anastomosed to minimize the impairment of cerebral blood flow. As the patient had a right dominant vertebral artery from the innominate artery, it was important to preserve it. The resected aneurysmal sac was sent to the pathology department for a final diagnosis, which indicated atherosclerosis with dystrophic calcification. Moreover, no specific bacteria were cultured from the aneurysmal sac.

Taking into consideration the patient's physical weakness because of her underlying disease, she was weaned from the ventilator only on day 6 after surgery and was moved out of the intensive care unit on day 10 after surgery. Although no neurological sequelae occurred, the patient's systemic condition was poor because of her long-standing chronic renal failure. As a result of a decrease in muscle strength due to prolonged bedridden status, she needed rehabilitation and was discharged in good condition 28 days after surgery. After discharge, the patient was in a healthy

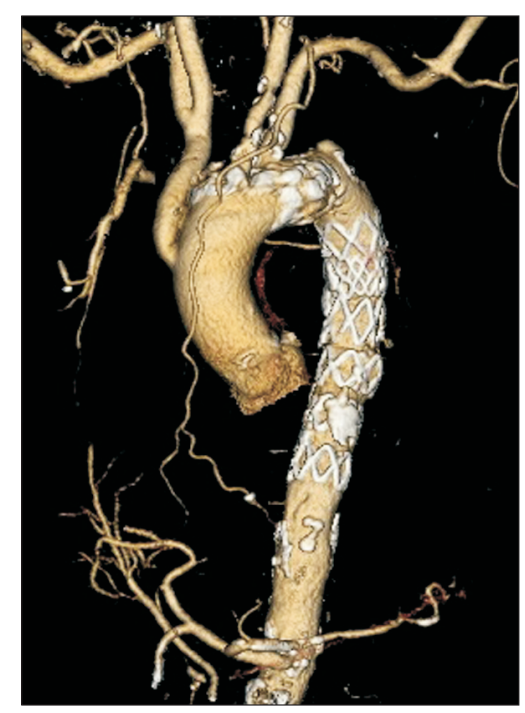

Fig. 3. Postoperative 3-dimensional computed tomography of the aorta. No unusual postoperative findings in the graft of the innominate artery and the stent graft of the descending thoracic aorta, such as leakage or stenosis, were found. 
state and underwent dialysis through the reintroduced brachio-axillary arteriovenous graft in the right arm, making outpatient visits to the departments of nephrology, infectious diseases, and cardiovascular surgery. Vancomycin was used intravenously for another 12 weeks after resection of the aneurysm on the advice of the department of infectious diseases, owing to the presence of gross pus during brachio-axillary arteriovenous graft removal and possible residual infection in the aneurysm of the descending aorta covered by the stent. Vancomycin was administered during dialysis after discharge.

With approval from the Institutional Review Board of Daegu Catholic University Medical Center (IRB approval no., CR-21-100) and a waiver of the requirement for individual informed consent, we reported our experience of successfully treating an innominate artery aneurysm using NIRS for cerebral monitoring without CPB support or hypothermic circulatory arrest.

\section{Discussion}

An innominate artery aneurysm is a rare and challenging condition. Studies have reported varying findings regarding the incidence of innominate aneurysms [1-3], but it is clear that they are very uncommon. Brewster et al. [3] reported that innominate aneurysms accounted for only 3 $(0.26 \%)$ of 1,147 aneurysms that were described in a case series.

Bacterial infection is one of the best-known causes of aneurysms, but recently, innominate artery aneurysms caused by infections have become less common than degenerative aneurysms. A study reported that atherosclerosis was the cause of more than $60 \%$ of innominate artery aneurysms [3,4]. Additionally, the incidence of aneurysms caused by autoimmune and connective tissue disorders and post-traumatic aneurysms has been increasing $[3,4]$.

Surgical treatment of an innominate artery aneurysm is necessary if the aneurysm is large and symptomatic or has ruptured. Kieffer et al. [4] described the indications of surgical treatment for innominate artery aneurysms and suggested that surgery should be considered in patients with good surgical risk if the aneurysm is saccular or its maximum transverse diameter is more than $3 \mathrm{~cm}$.

The methods of incision to approach the lesion are diverse. However, cervical incision, partial median sternotomy with cervical extension, or full median sternotomy with cervical extension can be used, depending upon the case. In the present case, we performed full median sternotomy using the cervical extension approach in prepara- tion for aneurysm rupture or the need for hypothermic circulatory arrest. Endovascular treatments have recently become available for several types of arterial or aortic aneurysms. Some studies have reported successful endovascular treatment of innominate artery aneurysms; however, careful selection of patients is necessary, and the long-term results of endovascular treatment have not yet been established.

Brain protection is one of the main concerns during surgery. Several authors have reported successful surgical treatment of innominate artery aneurysms using moderate to deep hypothermic circulatory arrest with or without selective cerebral perfusion [4]. However, hypothermic circulatory arrest makes the postoperative course challenging because of coagulopathy and the prolonged need for ventilator support $[5,6]$. Moreover, arrhythmia after hypothermic circulatory arrest is associated with considerable morbidity and mortality [5-7]. NIRS is now a widespread cerebral monitoring method used during carotid surgery. Various methods of monitoring cerebral perfusion can be employed, including transcranial Doppler, somatosensory-evoked potentials, and NIRS. NIRS has advantages over other methods of monitoring brain perfusion, as it is more practical and provides non-inferior protection of the brain from ischemia [8]. It is noninvasive, convenient, and easy to apply, and it may not require expert handling. Our center has successfully performed carotid endarterectomy using NIRS and reported excellent results [9]. A 20\% decrease in post-clamping cerebral oximetry values (compared with the baseline value) indicates the need for a shunt [10]. In the present case, a shunt or other forms of further perfusion were not necessary during right carotid artery clamping, as only approximately a $10 \%$ decrease in cerebral oximetry values was observed. We successfully performed surgery without any CPB support, complications such as stroke were not encountered, and brain perfusion was monitored with NIRS.

\section{Conflict of interest}

No potential conflict of interest relevant to this article was reported.

\section{Acknowledgments}

This work was supported by a grant from the Research Institute of Medical Science, Daegu Catholic University (2020). 


\section{ORCID}

Byeng Hun Jeon: https://orcid.org/0000-0003-4384-6703

Chul Ho Lee: https://orcid.org/0000-0002-9139-0619

Chi Hoon Bae: https://orcid.org/0000-0002-1481-2734

Jae Seok Jang: https://orcid.org/0000-0002-0693-8863

Jun Woo Cho: https://orcid.org/0000-0002-0786-9775

\section{References}

1. Regina G, Greco L, Fullone M, Testini M, Caruso G, Rizzi R. The emergency treatment of aneurysms of the supra-aortic trunks and of the internal carotid. Ann Ital Chir 2000;71:469-73.

2. Gay BB Jr, Walker JF. Aneurysm of the innominate artery; review of clinical and radiologic findings in 18 cases. Radiology 1953;60:80413.

3. Brewster DC, Moncure AC, Darling RC, Ambrosino JJ, Abbott WM. Innominate artery lesions: problems encountered and lessons learned. J Vasc Surg 1985;2:99-112.

4. Kieffer E, Chiche L, Koskas F, Bahnini A. Aneurysms of the innominate artery: surgical treatment of 27 patients. J Vasc Surg 2001;34: $222-8$

5. Ise H, Kitahara H, Oyama K, et al. Hypothermic circulatory arrest induced coagulopathy: rotational thromboelastometry analysis. Gen Thorac Cardiovasc Surg 2020;68:754-61

6. Lei Q, Chen L, Zhang Y, Fang N, Cheng W, Li L. Predictors of prolonged mechanical ventilation after aortic arch surgery with deep hypothermic circulatory arrest plus antegrade selective cerebral perfusion. J Cardiothorac Vasc Anesth 2009;23:495-500.

7. Pillarisetti J, Patel A, Bommana S, et al. Atrial fibrillation following open heart surgery: long-term incidence and prognosis. J Interv Card Electrophysiol 2014;39:69-75

8. Moritz S, Kasprzak P, Arlt M, Taeger K, Metz C. Accuracy of cerebral monitoring in detecting cerebral ischemia during carotid endarterectomy: a comparison of transcranial Doppler sonography, near-infrared spectroscopy, stump pressure, and somatosensory evoked potentials. Anesthesiology 2007;107:563-9.

9. Cho JW, Jang JS. Near-infrared spectroscopy versus transcranial doppler-based monitoring in carotid endarterectomy. Korean J Thorac Cardiovasc Surg 2017;50:448-52.

10. Zogogiannis ID, Iatrou CA, Lazarides MK, et al. Evaluation of an intraoperative algorithm based on near-infrared refracted spectroscopy monitoring, in the intraoperative decision for shunt placement, in patients undergoing carotid endarterectomy. Middle East J Anaesthesiol 2011;21:367-73. 